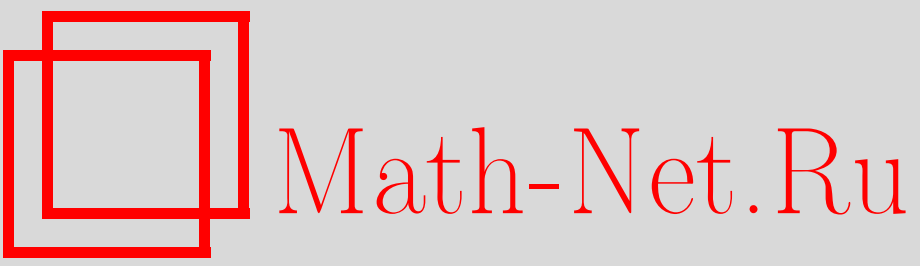

А. Е. Миронов, Спектральные подмногообразия главно поляризованного абелева многообразия, УМН, 2004, том 59, выпуск 5, 157-158

DOI: https://doi.org/10.4213/rm782

Использование Общероссийского математического портала Math-Net.Ru подразумевает, что вы прочитали и согласны с пользовательским соглашением

http://www.mathnet.ru/rus/agreement

Параметры загрузки:

IP: 34.239 .49 .27

26 апреля 2023 г., $16: 17: 29$ 


\title{
СПЕКТРАЛЬНЫЕ ПОДМНОГООБРАЗИЯ ГЛАВНО ПОЛЯРИЗОВАННОГО АБЕЛЕВА МНОГООБРАЗИЯ
}

\author{
А. Е. Миронов
}

В данной заметке построены коммутирующие матричные диффференциальные операторы по многим переменньм, отвечающие алгебраическим подмногообразиям в главно поляризованном абелевом многообразии, а также многомерный аналог иерархии Кадомцева-Петвиашвили (КП).

Обозначим через $X^{g}$ главно поляризованное абелево многообразие $\mathbb{C}^{g} /\left\{\mathbb{Z}^{g}+\Omega \mathbb{Z}^{g}\right\}$, где $\Omega$ симметричная комплексная матрица, $\operatorname{Im} \Omega>0$. Через $Y_{a_{j}}$ обозначим гладкую гиперповерхность, заданную уравнением

$$
\theta_{j}\left(z-a_{j}\right)=0, \quad a_{j} \in \mathbb{C}^{g},
$$

где $\theta_{j}(z)$ - тэта-функция степени $d_{j} \in \mathbb{N}$, т.е. аналитическая функция на $\mathbb{C}^{g}$ со свойством периодичности:

$$
\theta_{j}(z+\Omega m+n)=\exp \left(-d_{j} \pi i\langle m, \Omega m\rangle-2 d_{j} \pi i\langle m, z\rangle\right) \theta_{j}(z), \quad m, n \in \mathbb{Z}^{g} .
$$

Напомним, что пространство тэта-функций степени $d$ имеет размерность $d^{g}$. Любая алгебраическая гиперповерхность в $X^{g}$ задается как множество нулей некоторой тэта-функции подходящей степени. Гиперповерхность $Y_{a_{j}}$ можно также задать с помощью тэта-функций с характеристиками.

Через $Q$ обозначим гиперповерхность, заданную уравнением $\theta(z)=0$, где $\theta(z)$ - тэта-функция степени $d>d_{1}+\cdots+d_{k}$. Обозначим через $Y^{k}$ и $Q^{k}$ соответственно пересечения $Y_{a_{1}} \cap \cdots \cap Y_{a_{k}}$ и $Y^{k} \cap Q, k<g-1$. Будем предполагать, что многообразия $Y^{j}$ и $Q^{j}, j \leqslant k$, являются гладкими и неприводимыми и что $Y^{j}$ пересекается с $Y_{a_{j+1}}$ трансверсально. Предположим также, что $a_{j}$ находятся в общем положении, т.е. набор $\left(a_{1}, \ldots, a_{k}\right)$ принадлежит некоторому открытому всюду плотному подмножеству в $X^{g} \times \cdots \times X^{g}$.

Теорема 1. Существует вложение $L^{k}$ кольца мероморфных функций $\mathscr{A}^{k}$ на многообразии $Y^{k}$ с полюсом на $Q^{k}$ в кольцо $\left(N^{k} \times N^{k}\right)$-матричных дифференциальных операторов по $g-k$ переменным с аналитическими в окрестности нуля коэффициентами

$$
L^{k}: \mathscr{A}^{k} \rightarrow \operatorname{Mat}\left(N^{k}, g-k\right),
$$

число $N^{k}$ указано в формуле (1).

Существует семейство совместных собственных функций у операторов $L^{k}\left(\mathscr{A}^{k}\right)$, параметризованное точкамимногообразия $Y^{k}$, а именно, существует вектор-функция $\Psi(z, x), z \in Y^{k}$, с существенной особенностью на $Q^{k}$ такая, что

$$
L^{k}(\lambda) \Psi=\lambda \Psi, \quad \lambda \in \mathscr{A}^{k} .
$$

Для $\lambda, \mu \in \mathscr{A}^{k}$ операторы $L^{k}(\lambda)$ u $L^{k}(\mu)$ коммутируют.

Число $N^{k}$ вычисляется по формуле

$$
N^{k}=a_{1}^{k}+\cdots+a_{g-k+1}^{k},
$$

где

$$
\begin{gathered}
a_{n}^{k}=\mathscr{F}^{k}(n)-a_{1}^{k} C_{n-1+g-k}^{n-1}-\ldots-a_{n-1}^{k} C_{1+g-k}^{1}, \\
\mathscr{F}^{k}(n)=(d n)^{g}-\sum_{i}\left(d n-d_{i}\right)^{g}+\sum_{i, j}\left(d n-d_{i}-d_{j}\right)^{g}-\cdots+(-1)^{k}\left(d n-d_{1}-\ldots-d_{k}\right)^{g},
\end{gathered}
$$

$C_{j}^{i}-$ биномиальный коэффициент.

Работа вьполнена при поддержке Российского фонда фундаментальных исследований (гранты № НШ-2185.2003.1, 03-01-00403 и 03-01-06482). 
Многообразие $Y^{k}$ называется спектралшным. Из формулы присоединения и теоремы Лефшшеца о вложении вытекает, что $Y^{k}$ является многообразием общего типа.

Первые примеры коммутирующих дифференциальных операторов по многим переменным с матричными коэфффициентами построены Накаяшики [1], [2]. Спектральными многообразиями в этих примерах служат абелевы многообразия. Ротштейн [3] построил операторы, где в качестве спектрального многообразия рассматривается поверхность Фано. Теорема 1 доказывается с помощью методов работы [4], где был рассмотрен случай $d=d_{1}=\cdots=d_{k}$.

Имеется аналогия между спектральными данными обыкновенных коммутирующих дифференциальных операторов Берчналла-Чаунди-Кричевера [5] и спектральными данными операторов $L^{k}\left(\mathscr{A}^{k}\right)$ :

$$
\{\Gamma, D, P, f\} \longleftrightarrow\left\{Y^{k}, \mathscr{L}^{k}, Q^{k}, \lambda\right\},
$$

где $\Gamma$ - компактная риманова поверхность рода $g, D$ - неспециальный дивизор степени $g, P$ фиксированная точка на $\Gamma, f$-мероморфная функция на $\Gamma$ с полюсом в точке $P, \mathscr{L}^{k}-$ некоторое линейное расслоение над $Y^{k}$. Аналогом функции Бейкера-Ахиезера служит вектор-функция $\Psi$.

С каждым вложением $L^{k}$ связан аналог иерархии КП.

Теорема 2. Существует многомерный матричный аналог иерархии КП

$$
\left[L_{n}-\partial_{t_{n}}, L_{m}-\partial_{t_{m}}\right]=0
$$

где $L_{n}$ и $L_{m}-\left(N^{k} \times N^{k}\right)$-матричные дифференциальные операторы по переменным $x=\left(x_{1}, \ldots, x_{g-k}\right)$, коэффициенты которых зависят от $x$ и от $t=\left(t_{1}, t_{2}, \ldots\right)$.

Теоремш 1 и 2 могут быть обобщены на случай произвольного абелева многообразия, а также по схеме [4] на любой ранг $r>1$.

\section{СПИСОК ЛИТЕРАТУРЫ}

[1] A. Nakayashiki // Duke Math. J. 1991. V. 62. № 2. P. 315-358. [2] A. Nakayashiki // Amer. J. Math. 1994. V. 116. № 1. P. 65-100. [3] M. Rothstein // Duke Math. J. 1996. V. 84. № 3. Р. 565-598. [4] А. Е. Миронов // Сиб. матем. журн. 2002. Т. 43. № 5. С. 1102-1114. [5] И. М. Кричевер // УМН. 1977. Т. 32. № 6. С. 183-208.

Институт математики им. С. Л. Соболева СО РАН

Принято редколлегией

E-mail: mironov@math.nsc.ru

23.07.2004 